\title{
CONSTRAINING MODELS OF TWIN-PEAK QUASI-PERIODIC OSCILLATIONS WITH REALISTIC NEUTRON STAR EQUATIONS OF STATE
}

\author{
Gabriel TöröK ${ }^{1}$, Kateřina Goluchová ${ }^{1,2}$, Martin Urbanec ${ }^{1}$, Eva Šrámková ${ }^{1}$, Karel Adámek $^{1,2,3}$, Gabriela Urbancová ${ }^{1}$, \\ Tomáš Pecháčé ${ }^{1}$, Pavel Bakala ${ }^{1}$, ZdeněK Stuchlík ${ }^{2}$, Jiří Horák ${ }^{4}$, ANd Jakub Juryšek ${ }^{1,5}$ \\ ${ }^{1}$ Research Centre for Computational Physics and Data Processing, Institute of Physics, Faculty of Philosophy \& Science, Silesian University in Opava, \\ Bezručovo nám.13, CZ-746,01 Opava, Czech Republic; gabriel.torok@gmail.com, katka.g@ seznam.cz, martin.urbanec@physics.cz, eva.sramkova@fpf.slu.cz, \\ karel.adamek@fpf.slu.cz, gabi.urbancova@gmail.com, pechacek_t@seznam.cz, pavel.bakala@fpf.slu.cz, jakubjurysek@astronomie.cz \\ ${ }^{2}$ Research Centre for Theoretical Physics and Astrophysics, Institute of Physics, Faculty of Philosophy \& Science, Silesian University in Opava, \\ Bezručovo nám. 13, CZ-746, 01 Opava, Czech Republic; zdenek.stuchlik@ fpf.slu.cz \\ ${ }^{3}$ University of Oxford, Oxford e-Research Centre, 7 Keble Road, Oxford, OX1 3QG \\ ${ }^{4}$ Astronomical Institute, Bocni II 1401/2a, CZ-14131 Praha 4 - Sporilov, Czech Republic; hjirkoun@gmail.com \\ ${ }^{5}$ Astronomical Institute, Charles University Prague, Faculty of Mathematics and Physics, V Holešovičkách 2, Praha 8, CZ-180 00, Czech Republic. \\ Received 2016 January 11; revised 2016 October 26; accepted 2016 October 26; published 2016 December 21
}

\begin{abstract}
Twin-peak quasi-periodic oscillations (QPOs) are observed in the X-ray power-density spectra of several accreting low-mass neutron star (NS) binaries. In our previous work we have considered several QPO models. We have identified and explored mass-angular-momentum relations implied by individual QPO models for the atoll source $4 \mathrm{U}$ 1636-53. In this paper we extend our study and confront QPO models with various NS equations of state (EoS). We start with simplified calculations assuming Kerr background geometry and then present results of detailed calculations considering the influence of NS quadrupole moment (related to rotationally induced NS oblateness) assuming Hartle-Thorne spacetimes. We show that the application of concrete EoS together with a particular QPO model yields a specific mass-angular-momentum relation. However, we demonstrate that the degeneracy in mass and angular momentum can be removed when the NS spin frequency inferred from the X-ray burst observations is considered. We inspect a large set of EoS and discuss their compatibility with the considered QPO models. We conclude that when the NS spin frequency in 4U 1636-53 is close to $580 \mathrm{~Hz}$, we can exclude 51 of the 90 considered combinations of EoS and QPO models. We also discuss additional restrictions that may exclude even more combinations. Namely, 13 EOS are compatible with the observed twin-peak QPOs and the relativistic precession model. However, when considering the low-frequency QPOs and Lense-Thirring precession, only 5 EOS are compatible with the model.
\end{abstract}

Key words: accretion, accretion disks - equation of state - stars: neutron - X-rays: binaries

\section{INTRODUCTION}

Accreting neutron stars (NS) are believed to be the compact component in more than 20 low-mass X-ray binaries (LMXBs). In these systems, the mass is transferred from the companion by overflowing the Roche lobe and forming an accretion disk that surrounds the NS. The disk contributes significantly to the high X-ray luminosity of these objects, while most of the radiation comes from its inner parts and the disk-NS boundary layer. According to their X-ray spectral and timing properties, the NS LMXBs have been further classified into $\mathrm{Z}$ and atoll sources, whose names have been inspired by the shapes of the tracks they trace in the color-color diagram (e.g., van der Klis 2005). While the $\mathrm{Z}$ sources are generally more stable and brighter, the atoll sources are weaker and show significant changes in the X-ray luminosity. Both classes exhibit a variability over a wide range of frequencies. Except for irregular changes, their power spectra also contain relatively coherent features known as quasi-periodic oscillations (QPOs).

The so-called low-frequency QPOs have frequencies in the range of $1-100 \mathrm{~Hz}$. In the case of $\mathrm{Z}$ sources they have been further classified into horizontal, flaring, and normal branch oscillations (HBO, FBO, and NBO, respectively) depending on the position of the source in the color-color diagram. Oscillations of properties similar to HBOs have also been observed in several atoll sources (see van der Klis 2006, p. 39, for a review). Much attention among theoreticians is attracted to the kilohertz QPOs (100-1000 Hz), however, because their high frequencies are comparable to the orbital timescale in the vicinity of a NS. It is believed that this coincidence represents a strong indication that the corresponding signal originates in the innermost parts of the accretion disks or close to the surface of the NS itself. This belief has also been supported by means of the Fourier-resolved spectroscopy (e.g., Gilfanov et al. 2000).

The $\mathrm{kHz}$ QPOs have similar properties in both $\mathrm{Z}$ and atoll sources. They are frequently observed in pairs and are often called twin-peak QPOs. Their "upper" and "lower" QPO frequencies ( $\nu_{U}$ and $\nu_{L}$, respectively) exhibit a strong and remarkably stable positive correlation and clustering around the rational ratios. These ratios are emphasized either by the intrinsic source clustering, or by a weakness of the two QPOs outside the limited frequency range (suggesting a possible resonant energy exchange between two physical oscillators, Abramowicz et al. 2003a; Belloni et al. 2005, 2007; Barret \& Boutelier 2008; Török et al. 2008a, 2008b, 2008c; Horák et al. 2009; Boutelier et al. 2010). Other properties of each oscillation (e.g., the rms-amplitude and the quality factor) seem to mostly depend on its frequency, and the way in which they vary is different for the upper and lower oscillation. These differences often help to identify the type of $\mathrm{kHz}$ QPO in cases when only one peak is present in the power spectra (Barret et al. 2005, 2006; Méndez 2006; Török 2009).

Many models have been proposed to explain the rich phenomenology of twin-peak QPOs (Alpar \& Shaham 1985; Lamb et al. 1985; Miller et al. 1998; Psaltis et al. 1999; 
Table 1

Models Examined in this Work

\begin{tabular}{|c|c|c|}
\hline Model & Relations & $\nu_{L}-\nu_{U}$ Relation \\
\hline \multirow[t]{2}{*}{$\overline{\mathrm{RP}}$} & $\nu_{L}=\nu_{K}-\nu_{\mathrm{r}}$ & $\nu_{L}=\nu_{U}\left\{1-\left[1+\frac{8 j \nu_{U}}{\mathcal{F}-j \nu_{U}}-6\left(\frac{\nu_{U}}{\mathcal{F}-j \nu_{U}}\right)^{2 / 3}-3 j^{2}\left(\frac{\nu_{U}}{\mathcal{F}-j \nu_{U}}\right)^{4 / 3}\right]^{1 / 2}\right\}$ \\
\hline & $\nu_{U}=\nu_{K}$ & \\
\hline \multirow[t]{2}{*}{$\mathrm{TD}$} & $\nu_{L}=\nu_{K}$ & $\nu_{U}=\nu_{L}\left\{1+\left[1+\frac{8 j \nu_{L}}{\mathcal{F}-j \nu_{L}}-6\left(\frac{\nu_{L}}{\mathcal{F}-j \nu_{L}}\right)^{2 / 3}-3 j^{2}\left(\frac{\nu_{L}}{\mathcal{F}-j \nu_{L}}\right)^{4 / 3}\right]^{1 / 2}\right\}$ \\
\hline & $\nu_{U}=\nu_{K}+\nu_{\mathrm{r}}$ & \\
\hline WD & $\nu_{L}=2\left(\nu_{K}-\nu_{\mathrm{r}}\right)$ & $\nu_{U}=2 \nu_{K}-\nu_{\mathrm{r}}$ \\
\hline RP1 & $\nu_{L}=\nu_{K}-\nu_{\mathrm{r}}$ & $\nu_{U}=\nu_{\theta}$ \\
\hline $\mathrm{RP} 2$ & $\nu_{L}=\nu_{K}-\nu_{\mathrm{r}}$ & $\nu_{U}=2 \nu_{K}-\nu_{\theta}$ \\
\hline
\end{tabular}

Wagoner 1999; Abramowicz \& Kluźniak 2001; Kato 2001, 2007, 2008; Kluźniak \& Abramowicz 2001; Wagoner et al. 2001; Titarchuk \& Wood 2002; Abramowicz et al. 2003b, 2003c; Rezzolla et al. 2003; Kluźniak et al. 2004; Bursa 2005; Pétri 2005; Zhang 2005; Török et al. 2007, 2016; Čadež et al. 2008; Stuchlík et al. 2008; Germanà et al. 2009; Kostić et al. 2009; Mukhopadhyay 2009; Stuchlík et al. 2013, 2014, 2015; Wang et al. 2015, and several others). While any acceptable model should address both the excitation mechanism and subsequent modulation of the resulting X-ray signal as well as their overall observational properties, most of the theoretical effort has so far been devoted to the observed frequencies. Clearly, their correlations serve as a first test of the model viability.

\subsection{Aims and Scope of this Paper}

Comparison between the observed and the expected frequencies can reveal the mass and angular momentum of the NS. These can be confronted with models of rotating NS based on a modern equation of state (EoS, e.g., Urbanec et al. 2010b). In Török et al. (2012) we have identified and explored mass-angular-momentum relations implied in Kerr spacetimes by individual QPO models. We have also discussed that the degeneracy in mass and angular momentum can be removed when the NS spin frequency is known.

Here we extend our study and confront QPO models with a large set of NS EoS while focusing on the influence of NS quadrupole moment that is related to its rotationally induced oblateness. The paper is arranged as follows. In Section 2 we very briefly recall individual QPO models that we consider together with previously obtained results. We present here the completed simplified calculations that assume Kerr background geometry and the atoll source $4 \mathrm{U} 1636-53$. These follow previous comparison between predictions of the relativistic precession (RP) model and $5 \mathrm{EoS}$. The consideration is extended to other models and a large set of 18 EoS. Sections 3.1 and 4 bring detailed consequent calculations of RP model predictions considering the influence of the NS quadrupole moment within Hartle-Thorne spacetimes. We show here that the application of concrete Sly 4 EoS within the model in Hartle-Thorne spacetime brings a specific mass-spin relation. This relation is confronted with the NS spin frequency inferred from the X-ray burst observations. In Section 5 we present analogical results for the whole set of 5 QPO models and 18 EoS and outline their implications. We also discuss here the implications of the consideration of low-frequency QPOs.

\section{TWIN-PEAK QPO MODELS APPROXIMATED IN KERR SPACETIMES}

Within the framework of many QPO models, the observable frequencies can be expressed directly in terms of epicyclic frequencies. Formulae for the geodesic Keplerian and radial and vertical epicyclic frequencies in Kerr spacetimes were first derived by Aliev \& Galtsov (1981). In a commonly used form (e.g., Török \& Stuchlík 2005) they read

$$
\Omega_{K}=\frac{\mathcal{F}}{j+x^{3 / 2}}, \quad \nu_{\mathrm{r}}=\Gamma \Omega_{K}, \quad \nu_{\theta}=\Delta \Omega_{K},
$$

where

$$
\begin{aligned}
\Gamma & =\sqrt{\frac{-3 j^{2}+8 j \sqrt{x}+(-6+x) x}{x^{2}}}, \\
\Delta & =\sqrt{1+\frac{j(3 j-4 \sqrt{x})}{x^{2}}},
\end{aligned}
$$

$x \equiv r / M$, and the "relativistic factor" $\mathcal{F}$ reads $\mathcal{F} \equiv c^{3} /(2 \pi G M)$. We note that Kerr geometry represents an applicable approximation of NS spacetimes when the mass of the compact object is high (Török et al. 2010; Urbanec et al. 2013).

The above formulae are valid for Kerr spacetimes and describe (epicyclic) slightly perturbed circular geodesic motion well. This description of epicyclic motion of test particles that is relevant to standard thin accretion disks may also well approximate epicyclic motion in fluid accretion flow provided that the pressure effects in the fluid are negligible and linear quasi-incompressible modes are considered. Formulae for geodesic epicyclic oscillations are often assumed within several QPO models based on accretion disk hot-spot as well as global fluid motion (e.g., Stella \& Vietri 1999, 2001; Abramowicz \& Kluźniak 2001; Kluźniak \& Abramowicz 2002). Here we investigate a subset of models that have previously been considered in the study of Török et al. (2012).

\subsection{Individual Models of QPOs and their Predictions}

The RP model explains the $\mathrm{kHz}$ QPOs as a direct manifestation of modes of relativistic epicyclic motion of blobs at various radii $r$ in the inner parts of the accretion disk (Stella \& Vietri 1999). For the RP model, one can easily solve relations defining the upper and lower QPO frequencies in terms of the orbital frequencies and arrive at an explicit formula that relates the upper and lower QPO frequencies in units of 
a)

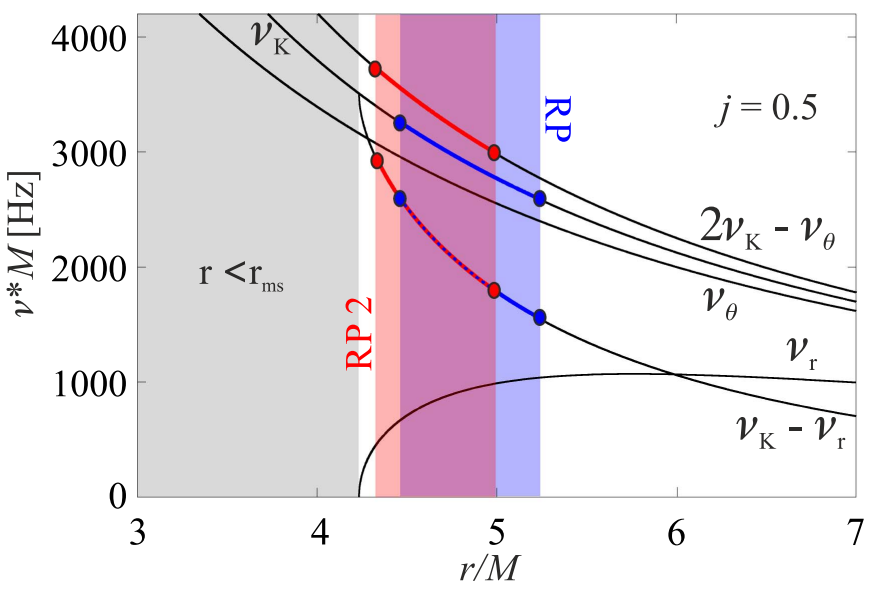

c)

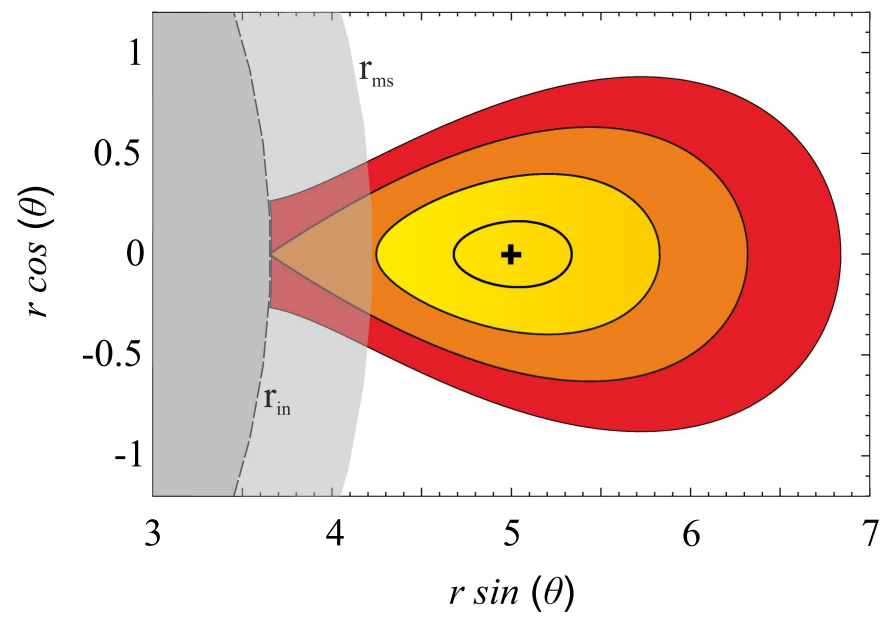

b)


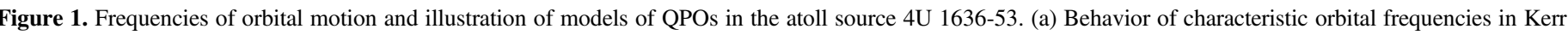



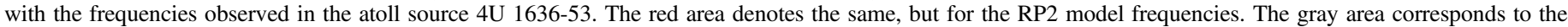

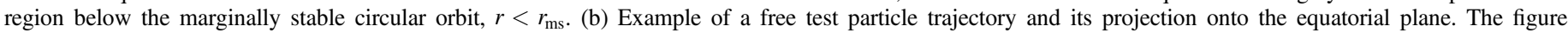

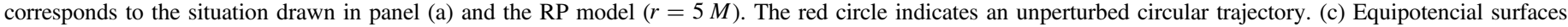

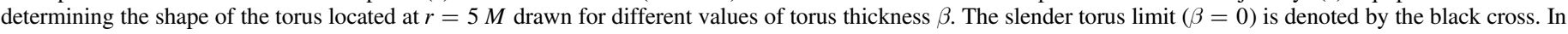

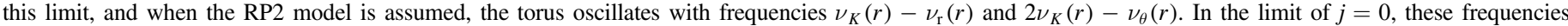

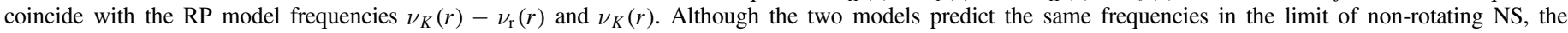
associated physical mechanisms are not the same.

Hertz (Török et al. 2010). We show this relation in Table 1. The concept of the tidal disruption (TD) model is similar to the RP model, but the QPOs are atributed to a disruption of large accreting inhomogenities (Germanà et al. 2009). The explicit relation between the two observed QPO frequencies can be evaluated in a way similar to the case of the RP model (Török et al. 2012), and we also include this relation in Table 1.

While the former two models assume motion of a hot-spot propagating within the accretion disk, the warp disk (WD) model assumes non-axisymmetric oscillation modes in a thick disk (Kato 2001). The two other considered models, RP1 and RP2, also deal with non-axisymmetric disk-oscillation modes. The frequencies of these modes coincide with the frequencies predicted by the RP model in the limit of $j=0$ (Bursa 2005; Török et al. 2010). Although the relevant frequencies coincide in the case of non-rotating NS, they correspond to a different physical situation (see Figure 1 for an illustration). We include the expressions for lower and upper QPO frequency for all the three disk-oscillation models in Table 1.

Török et al. (2010, 2012) assumed a high-mass (Kerr) approximation of NS spacetimes and relations from Table 1. We have demonstrated that for each twin-peak QPO model and a given source, the model consideration results in a specific relation between the NS mass $M$ and angular momentum $j$ rather than in their single preferred combination. We payed special attention to the atoll source $4 \mathrm{U} 1636-53$ and evaluated mass-angular-momentum relations for all discussed QPO models. ${ }^{6}$

\subsection{Twin-Peak QPO Models versus NS EoS}

Török et al. (2012) compared a $\chi^{2}$ map describing the quality of the RP model fit of the $4 \mathrm{U} 1636-53$ data to the $M-j$ relations implied by five specific NS EoS. These $M-j$ relations were calculated assuming that the NS spin frequency $\nu_{\mathrm{S}}$ is $580 \mathrm{~Hz}$ (Strohmayer \& Markwardt 2002; Galloway et al. 2008; Watts 2012). In these calculations we used the approach

\footnotetext{
6 Lin et al. (2011) have performed a similar analysis assuming a different set of twin-peak QPO frequency data points for the atoll source 4U 1636-53. The data points in their study have been obtained via common processing of a large amount of data, while the data points used by Török et al. (2012) correspond to individual continuous observations of the source. It was shown in Török et al. (2012) that the results of the two studies are consistent (see also the NS parameteres resulting within the two studies denoted in Figure 2 and in Török et al. 2016).
} 


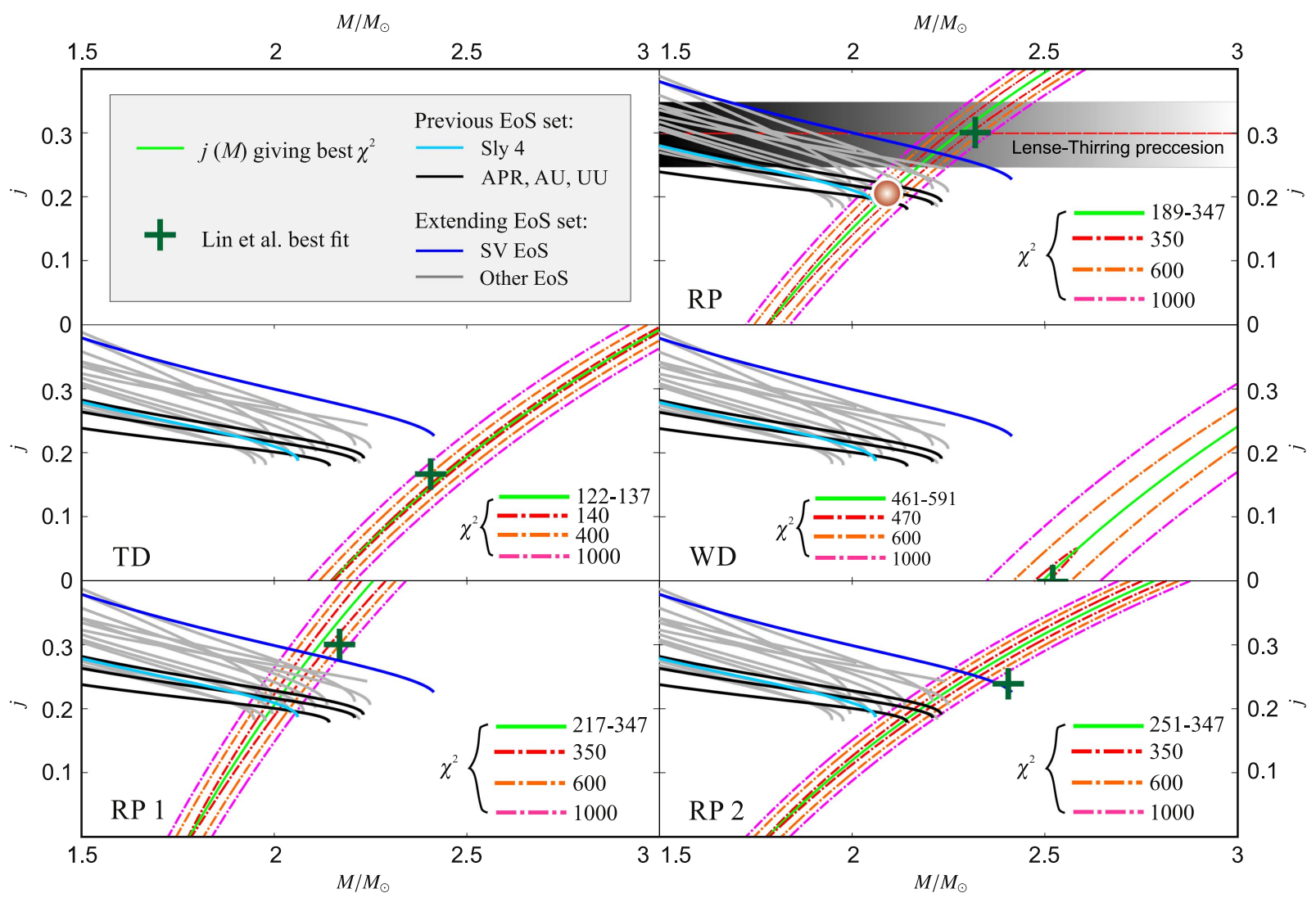

Figure 2. $\chi^{2}$ maps (20d.o.f.) calculated from data of the atoll source $4 \mathrm{U}$ 1636-53 and individual QPO models within Kerr spacetimes vs. mass-angular-momentum relations predicted by NS EoS. For the calculations we consider 14 more EOS in addition to 4 EoS (SLy 4, APR, AU-WFF1, and UU-WFF2) assumed in Török et al. (2012). The full set of $18 \mathrm{EoS}$ is listed in Table 2. In each panel the green line indicates the best $\chi^{2}$ for a fixed $M$, while the dashed green line denotes its quadratic approximation. The white lines indicate the corresponding $1 \sigma$ and $2 \sigma$ confidence levels. The NS EoS are assumed for the rotational frequency of $580 \mathrm{~Hz}$ inferred from the X-ray burst observations. The green crosses denote the mass and angular momentum combinations reported for 4U 1636-53 and individual QPO models by Lin et al. (2011). The red spot roughly indicates the combination of mass and spin inferred from the common consideration of the RP model, NS spin frequency of $580 \mathrm{~Hz}$, and $4 \mathrm{EoS}$ as discussed by Török et al. (2012). The horizontal dashed red line together with the horizontal shaded bar indicate additional restrictions on the RP model that follow from consideration of Lense-Thirring precession, as discussed in Török et al. (2012).

of Hartle (1967), Hartle \& Thorne (1968), Chandrasekhar \& Miller (1974), Miller (1977), and Urbanec et al. (2010a).

In the top panel of Figure 2 we show a comparison between predictions of the RP model and $4 \mathrm{EoS}$ carried out in Török et al. (2012). We note that the choice of concrete EoS used within that paper was motivated by low values of a scaled quadrupole moment $\tilde{q} \equiv q / j^{2}$ of the assumed NS configurations. ${ }^{7}$ Although QPO model predictions are drawn for simplified calculations assuming Kerr background geometry, in following we do not restrict ourselves to high-mass (compactness) NS. We thus add 14 more EoS, which are indicated within the figure. The full set of $18 \mathrm{EoS}$ considered hereafter is listed in Table 2. In the other panels of Figure 2 we make the same comparison, but for the other four considered QPO models.

Török et al. (2012) directly compared (a few) EoS and the RP model. Inspecting our overall extended Figure 2, we can expect that QPO models place strong restrictions on NS parameters and EoS, or vice versa. For instance, a direct confrontation of EoS and TD model predictions strongly suggests that the model (favored within the study of Lin

\footnotetext{
Török et al. (2012) also assumed one more EOS (WS, Wiringa et al. 1988 Stergioulas \& Friedman 1995). We do not consider this EOS here since it does not fulfill the requirements of the current observational tests.
}

et al. 2011) entirely fails to meet the requirements given by the consideration of NS EoS. Moreover, by comparing overlaps between the RP model relation and curves denoting the requirements of individual EoS, other interesting information can be obtained: there is a difference between overlaps considered in Török et al. (2012), which are denoted here by the red spot in Figure 2, and overlaps given by the newly considered EoS. Clearly, the high quadrupole moment of NS configurations related to the latter set of EoS increases the required NS angular momentum. For instance, there is $j \doteq 0.19$ for Sly 4 versus $j \doteq 0.28$ for SV EoS. It is also apparent that this effect can be important for the consideration of Lense-Thirring precession and low-frequency QPOs within the framework of the RP model.

Motivated by these findings, below we explore restrictions on QPO models in detail and perform consistent calculations in Hartle-Thorne spacetimes.

\section{CALCULATIONS IN HARTLE-THORNE SPACETIMES}

So far, we have considered only a Kerr approximation of the rotating NS spacetime assuming that the star is very compact. In this case the NS quadrupole moment $q$ related to its rotationally induced oblateness reaches low values, and we have $\tilde{q} \approx 1$. In a more general case of $\tilde{q}>1$, one should 
Table 2

EoS Examined in this Work

\begin{tabular}{llccc}
\hline \hline EoS & $M_{\max }$ & $\begin{array}{c}R \\
(\mathrm{~km})\end{array}$ & $\begin{array}{c}n_{\mathrm{c}} \\
{\left[[\mathrm{fm}]^{-3}\right)}\end{array}$ & References \\
\hline Sly 4 & 2.04 & 9.96 & 1.21 & 1 \\
SkI5 & 2.18 & 11.3 & 0.97 & 1 \\
SV & $2.38^{*}$ & 11.9 & 0.80 & 1 \\
SkO & 1.97 & 10.3 & 1.19 & 1 \\
Gs & 2.08 & 10.8 & 1.07 & 1 \\
SkI2 & 2.11 & 11.0 & 1.03 & 1 \\
SGI & 2.22 & 10.9 & 1.01 & 1 \\
APR & 2.21 & 10.2 & 1.12 & 2 \\
AU & 2.13 & 9.39 & 1.25 & 3 \\
UU & 2.19 & 9.81 & 1.16 & 3 \\
UBS & $2.20 *$ & 12.1 & 0.68 & 4 \\
GLENDNH3 & 1.96 & 11.4 & 1.05 & 5 \\
Gandolfi & 2.20 & 9.82 & 1.16 & 6 \\
QMC700 & 1.95 & 12.6 & 0.61 & 7 \\
KDE0v1 & 1.96 & 9.72 & 1.29 & 8 \\
NRAPR & 1.93 & 9.85 & 1.29 & 9 \\
PNM L80 & 2.02 & 10.4 & 1.16 & 10 \\
J35 L80 & 2.05 & 10.5 & 1.14 & 10 \\
\hline
\end{tabular}

Note. The individual columns indicate the maximum mass and corresponding radius, and the central baryon number density for each EoS along with the relevant references. The asterisks mark three EoS whose maximum mass corresponds to the maximum density allowed by the available EoS table, and not to a marginally stable star.

References. (1) Rikovska Stone et al. (2003), (2) Akmal et al. (1998), (3) Wiringa et al. (1988), (4) Urbanec et al. (2010a), (5) Glendenning (1985), (6) Gandolfi et al. (2010), (7) Rikovska Stone et al. (2007), (8) Agrawal et al. (2005), (9) Steiner et al. (2005), (10) Newton et al. (2013).

assume NS spacetime approximated by the Hartle-Thorne geometry (Hartle 1967; Hartle \& Thorne 1968). ${ }^{8}$

Based on the Hartle-Thorne approximation, the Keplerian orbital frequency can be expressed as (Abramowicz et al. 2003a)

$$
\Omega_{K}=\frac{\mathcal{F}}{x^{3 / 2}}\left[1-\frac{j}{x^{3 / 2}}+j^{2} F_{1}(x)+q F_{2}(x)\right],
$$

where

$$
\begin{aligned}
F_{1}(x)= & {\left[48-80 x+4 x^{2}-18 x^{3}+40 x^{4}+10 x^{5}\right.} \\
& \left.+15 x^{6}-15 x^{7}\right]\left(16(x-2) x^{4}\right)^{-1}+A(x), \\
F_{2}(x)= & \frac{5\left(6-8 x-2 x^{2}-3 x^{3}+3 x^{4}\right)}{16(x-2) x}-A(x), \\
A(x)= & \frac{15\left(x^{3}-2\right)}{32} \ln \left(\frac{x}{x-2}\right) .
\end{aligned}
$$

The radial and vertical epicyclic frequencies are then described by the following terms

$$
\nu_{\mathrm{r}}^{2}=\frac{\mathcal{F}^{2}(x-6)}{x^{4}}\left[1+j H_{1}(x)-j^{2} H_{2}(x)-q H_{3}(x)\right],
$$

\footnotetext{
8 The adopted approximation represents a convenient alternative to a (more precise) numerical approach (discussed in the same context by Stella et al. 1999) or other spacetime descriptions (e.g., Manko et al. 2000; Stute \& Camenzind 2002; Pappas 2015), see also Bonazzola et al. (1993, 1998), Stergioulas \& Friedman (1995), Nozawa et al. (1998), Ansorg et al. (2003), and Berti et al. (2005).
}

$$
\nu_{\theta}^{2}=\frac{\mathcal{F}^{2}}{x^{3}}\left[1-j G_{1}(x)+j^{2} G_{2}(x)+q G_{3}(x)\right]
$$

where

$$
\begin{aligned}
H_{1}(x)= & \frac{6(x+2)}{x^{3 / 2}(x-6)}, \\
H_{2}(x)= & {\left[8 x^{4}(x-2)(x-6)\right]^{-1}\left[384-720 x-112 x^{2}-76 x^{3}\right.} \\
& \left.-138 x^{4}-130 x^{5}+635 x^{6}-375 x^{7}+60 x^{8}\right]+C(x), \\
H_{3}(x)= & \frac{5\left(48+30 x+26 x^{2}-127 x^{3}+75 x^{4}-12 x^{5}\right)}{8 x(x-2)(x-6)} \\
& -C(x), \\
C(x)= & \frac{15 x(x-2)\left(2+13 x-4 x^{2}\right)}{16(x-6)} \ln \left(\frac{x}{x-2}\right), \\
G_{1}(x)= & \frac{6}{x^{3 / 2},} \\
G_{2}(x)= & {\left[8 x^{4}(x-2)\right]^{-1}\left[48-224 x+28 x^{2}+6 x^{3}-170 x^{4}\right.} \\
& +295 x^{5} \\
& \left.-165 x^{6}+30 x^{7}\right]-B(x), \\
8 x(x-2) & 5\left(6+34 x-59 x^{2}+33 x^{3}-6 x^{4}\right) \\
G_{3}(x)= & \frac{5(x),}{16} \ln \left(\frac{x-2}{x-2}\right) . \\
B(x)= & \frac{15(2 x-1)(x-2)^{2}}{3}(x)
\end{aligned}
$$

\subsection{Results for the RP Model}

Assuming the above formulae, we have calculated $3 \mathrm{D}-\chi^{2}$ maps for the RP model. In the left panel of Figure 3 we show the behavior of the best $\chi^{2}$ as a function of $M$ and $j$ for several color-coded values of $\tilde{q}$. For each value of $\tilde{q}$ there is a preferred $M-j$ relation. We find that, although such a relation has a global minimum, the gradient of $\chi^{2}$ along the relation is always much lower than the gradient in the perpendicular direction. In other words, $\chi^{2}$ maps for a fixed $\tilde{q}$ are of the same type as the maps calculated in the Kerr spacetime. It then follows that there is a global $M-j-\tilde{q}$ degeneracy in the sense discussed by Török et al. (2012); see their Figure 3.

As emphasized by Urbanec et al. (2010b), Török et al. (2010), Kluźniak \& Rosińska (2013), Török et al. (2014), Rosińska et al. (2014), and Boshkayev et al. (2015), Newtonian effects following from the influence of the quadrupole moment act on the orbital frequencies in a way opposite to that which is related to relativistic effects following from the increase of the angular momentum. The behavior of the relations shown in the left panel of Figure 3 is determined by this interplay. Because of this, we can see that the increased NS quadrupole moment can compensate for the increase in estimated mass given by a high angular momentum.

\section{CONSIDERATION OF NS MODELS GIVEN BY CONCRETE EOS}

The relations for the RP model drawn in the left panel of Figure 3 result from fitting of the 4U 1636-53 data points considering the general Hartle-Thorne spacetime. The consideration does not include strong restrictions on spacetime properties following from NS modeling based on present EoS. It can be shown that a concrete NS EoS covers only a 2D 

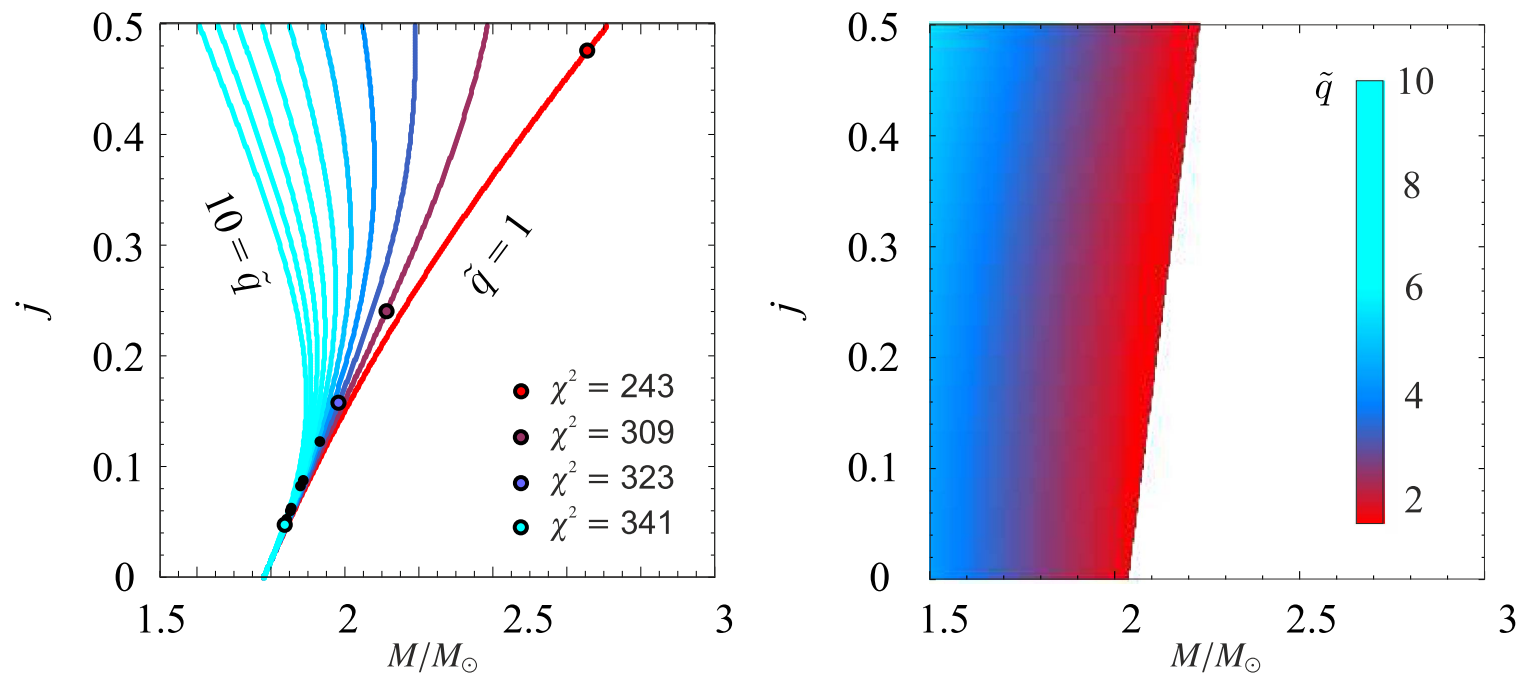

Figure 3. Left: behavior of the best $\chi^{2}$ as a function of $M$ and $j$ for several values of $\tilde{q}$. The dots denote global minima for each value of $\tilde{q}$ (see, however, the main text, Section 3.1, for a comment on this). Right: the 2D surface in the 3D $M-j-\tilde{q}$ space given by the SLy 4 EoS.
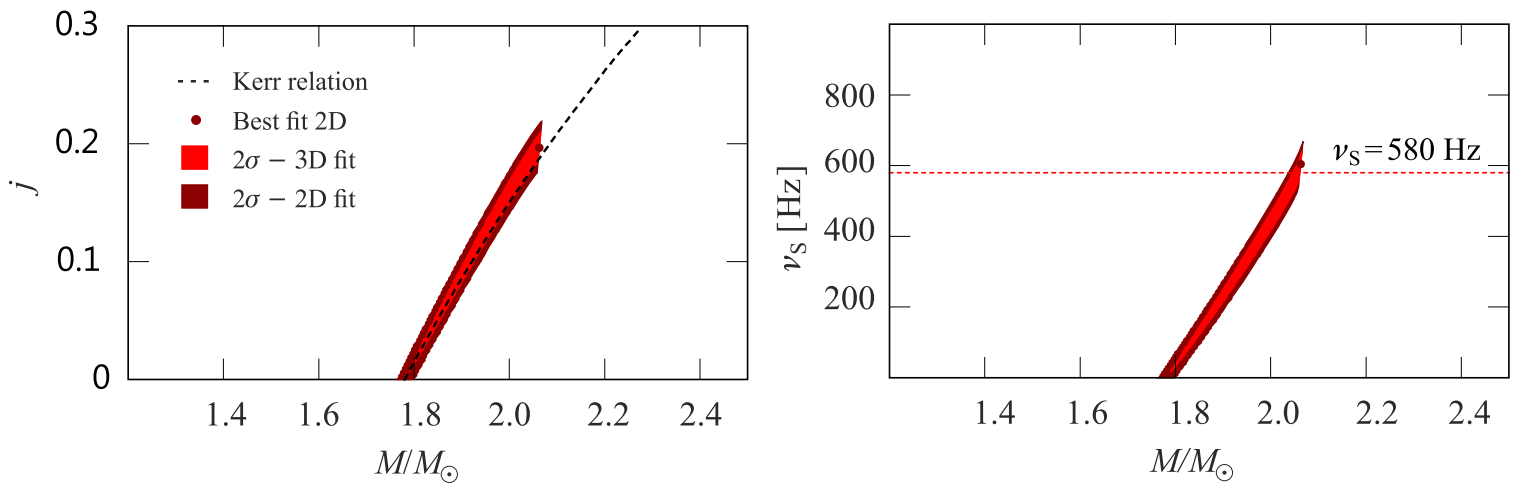

Figure 4. Left: the final $M-j$ map implied by the RP model and the Sly 4 EoS. The light color area denotes an intersection between the 2D surface given by the Sly 4 EoS and $3 \mathrm{D}$ volume corresponding to the $2 \sigma$ confidence level given by the RP best-fit model found in the intervals of $M \in\left[1 M_{\odot}, 4 M_{\odot}\right], j \in[0.0,0.5]$, and $\tilde{q} \in[1,10]$. The $\chi^{2}$ minimum of 303/20d.o.f. at the 2D surface is denoted by the dark marker. The dark color area denotes the $2 \sigma$ confidence level calculated when this local minimum is assumed as a global minimum, provided that the QPO frequency error bars are underestimated by a corresponding factor $\xi_{2 \mathrm{D}}=3.9$. We can see that in this particular case, there is almost no difference between the two areas. The dashed curve indicates the $M-j$ relation obtained from the simplified consideration of Kerr spacetimes (see Section 2). Right: the same map, but drawn for the NS spin frequency $\nu_{\mathrm{S}}$. The horizontal dashed red line denotes the spin frequency measured from the X-ray bursts (i.e., $\nu_{\mathrm{S}}=580 \mathrm{~Hz}$ ). The minimum of $\chi^{2}$ for the spin $\nu_{\mathrm{S}}=580 \mathrm{~Hz}$ corresponds to $\chi^{2}=305 / 21$ d.o.f.

surface in the 3D $M-j-\tilde{q}$ space since the quadrupole moment is determined by rotationally induced NS oblateness. Thus, when a given EoS is assumed, only the corresponding 2D surface is relevant for fitting data points by a given QPO model. Following Urbanec et al. (2013), we illustrate such a surface in the right panel of Figure 3 for the SLy 4 EoS. The color-coding of the plot is the same as the one in the left panel of the same figure.

The final $M-j$ map for the RP model and Sly 4 EoS, i.e., the values of $M$ and $j$ implied by the common consideration of the two panels of Figure 3, is shown in the left panel of Figure 4. The right panel of this figure then shows an equivalent map drawn for the NS mass and spin frequency $\nu_{\mathrm{S}}$.

\subsection{NS Mass Inferred Assuming X-Ray Burst Measurements}

The left panel of Figure 4 shows that the concrete EoS, SLy 4 , considered for the RP model implies a clear $M-j$ relation. This relation exhibits only a shallow $\chi^{2}$ minimum. The right panel of the same figure shows the equivalent relation between the NS mass and the spin frequency as well as its shallow minimum. Taking into account the spin frequency inferred from the X-ray bursts, $580 \mathrm{~Hz}$, we can find from Figure 4 that the NS mass and angular momentum have to take values of

$$
M=(2.06 \pm 0.01) M_{\odot}, \quad j \doteq 0.2 .
$$

These values are in a good agreement with those inferred from the simplified consideration using Kerr spacetimes (see Figure 2). Considering the shallow $\chi^{2}$ minima denoted in Figure 4, it may be interesting that its frequency value almost coincides with the measured spin frequency of $580 \mathrm{~Hz}$.

\section{DISCUSSION AND CONCLUSIONS}

In addition to the Sly 4 EoS, we have investigated a wide set of 17 other EoS that are based on different theoretical models. All these EoS are listed in Table 2, where we show the maximum NS mass allowed by each EoS as well as the corresponding NS radius and the central number density. All these EoS are compatible with the highest observed NS masses 


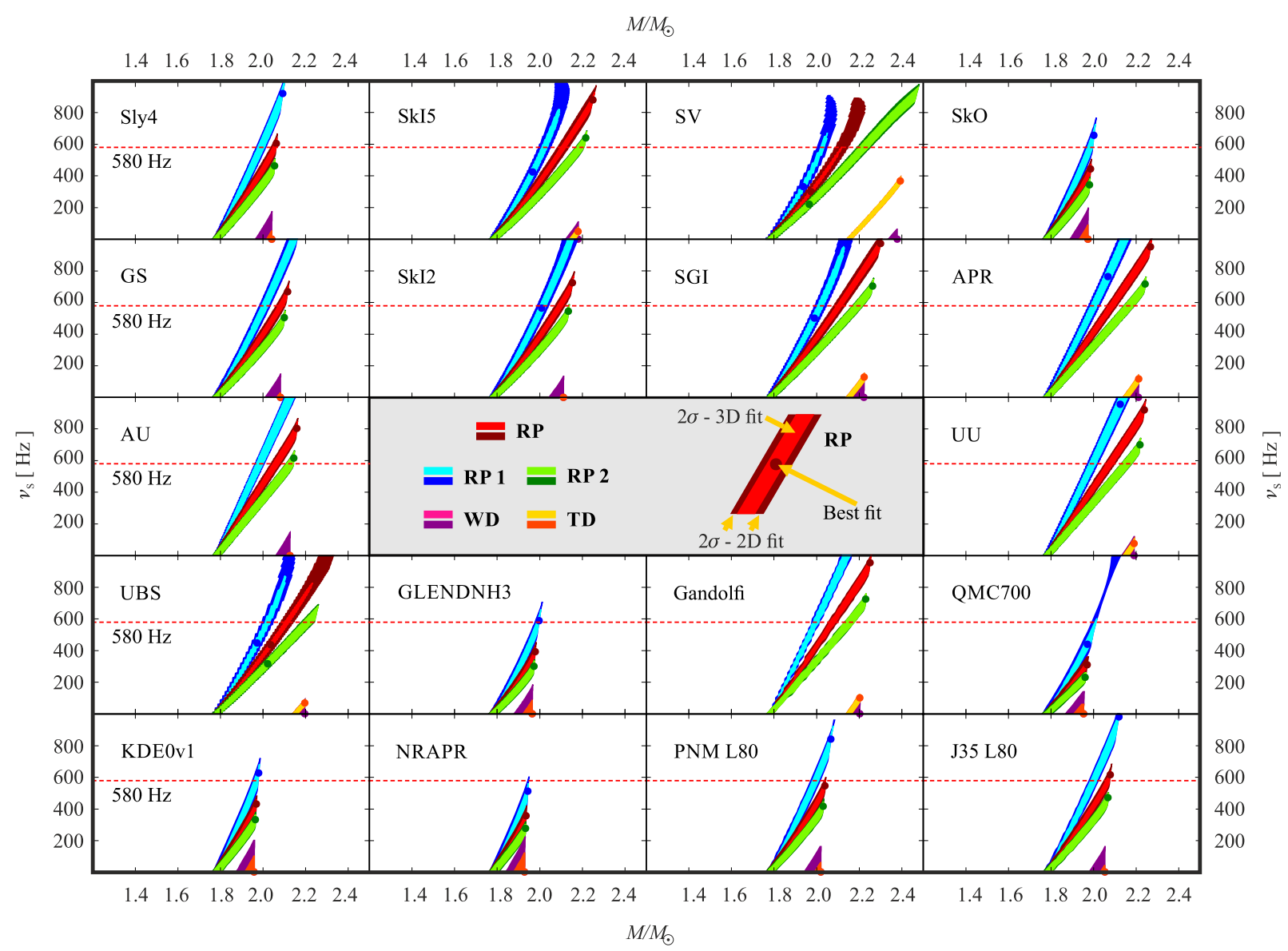

Figure 5. Mass-spin maps for the considered QPO models and 18 different EoS. The light color area denotes an intersection between the 2D surface given by the EoS and 3D volume corresponding to $2 \sigma$ confidence level associated to the best fit for a given model and the intervals of $M \in\left[1 M_{\odot}, 4 M_{\odot}\right], j \in[0.0,0.5]$, and $\tilde{q} \in[1,10]$. The $\chi^{2}$ minimum at the 2D surface is denoted by the dark marker. The dark color area denotes the $2 \sigma$ confidence level calculated when this local minimum is assumed as a global one providing that the QPO frequency error bars are underestimated by a corresponding factor $\xi_{2 \mathrm{D}}$. The horizontal dashed red lines denote spin frequency measured from the X-ray bursts (i.e., $\nu_{\mathrm{S}}=580 \mathrm{~Hz}$ ).

(see, e.g., Klähn et al. (2006), Steiner et al. (2010, 2015), Klähn et al. (2007), Dutra et al. (2012), and Dutra et al. (2014) for various tests of EoS and their applications, and Demorest et al. (2010) and Antoniadis et al. (2013) for the highest observed NS masses).

In Figure 5 we show several relations between the mass and spin frequency obtained for the RP model and our large set of EoS. These relations are similar to those implied by the Sly 4 EoS discussed above. However, we can see that in several cases, a given EoS does not provide any match for the NS spin of $580 \mathrm{~Hz}$. This can rule out the combination of the considered RP model and given specific EoS. The selection effect comes from the correlation between the estimated mass and angular momentum and the limits on maximal mass allowed by the individual EoS.

\subsection{Selecting Combinations of QPO Models and EoS}

We also found an analogical selection effect for the other four examined QPO models. The corresponding $M-j$ maps are shown in Figure 5. The results for all considered models are summarized in Table 3. The table shows which of the models and EoS are compatible, and which of them are not. Overall, there are 39 matches from the 90 investigated cases for the NS spin frequency of $580 \mathrm{~Hz}$. We can therefore conclude that for the NS spin frequency in $4 \mathrm{U} 1636-53$ to be close to $580 \mathrm{~Hz}$, we can exclude 51 of the 90 considered combinations of EoS and
QPO models. This result follows from the requirement of the relatively high masses implied by the individual QPO models and from the increase in these masses with the NS spin.

\subsection{Implications for $Q P O$ Models}

When we assume that the Hartle-Thorne geometry is restricted to the range of angular momentum and scaled quadrupole moment $\{j, \tilde{q}\} \in\{[0,0.4],[1,10]\}$, the four investigated QPO models imply a relatively broad range of NS mass, $M \in[1.6,3.4] M_{\odot}\left(M \in[1.8,2.5] M_{\odot}\right.$ when $\left.j=0\right)$. In Figure 6 we illustrate a corresponding comparison between the data and some individual fits. From inspecting Figure 6, we can see that the quality of the fits is rather poor (represented by $\chi^{2} /$ d.o.f. $\sim 10$, see Table 3 ). The comparison between data and curves drawn for the RP model indicates the possible presence of systematic errors within the model. This also holds for the RP1, RP2, and WD model. The trend is somewhat better only in the case of the TD model. This has also been noted by Lin et al. (2011). However, when we take into account requirements given by present EoS and the NS spin of $580 \mathrm{~Hz}$, the TD model is ruled out (see the green curve in the bottom right panel of Figure 6). The range of NS mass that corresponds to the considered models is then reduced to $M \in[2.0,2.2] M_{\odot}$.

Remarkably, the consideration of Hartle-Thorne spacetime does not improve the quality of the fits. For instance, the deviation of the RP model curve from the data discussed by Lin 
Table 3

Results for the Considered EoS and QPO Models

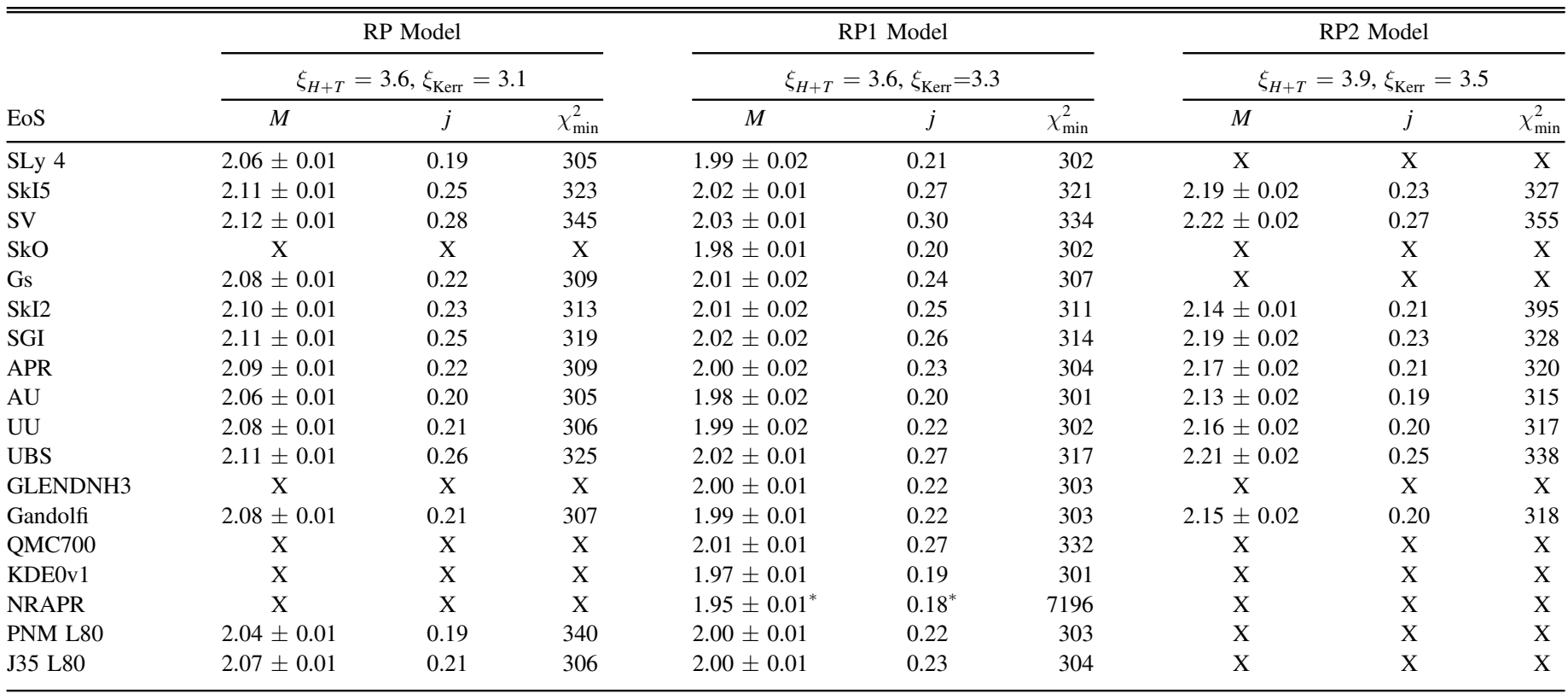

Note. Values of $\xi=\sqrt{\chi_{\min }^{2} / \text { dof }}$ corresponding to global minima of $\chi_{\min }^{2}$ in Hartle-Thorne spacetime are compared to values obtained for Kerr spacetimes in Török et al. (2012). Asterisks denote that the indicated values of $M$ and $j$ are connected to the dark color area in Figures 4 and 5 . In this case there is no intersection between the spin frequency curve in the 2D EoS plane and the $2 \sigma$ level 3D volume around the global minima of the QPO model fit in the Hartle-Thorne spacetime. The local minimum, $\chi_{2 \mathrm{Dmin}}^{2}$, at the $2 \mathrm{D}$ EoS plane is then assumed as a global minimum provided that the QPO frequency error bars are underestimated by a corresponding factor, $\xi_{2 \mathrm{D}}=\sqrt{\chi_{2 \mathrm{Dmin}}^{2} / \mathrm{dof}}$. The $X$-symbol indicates that the spin frequency $580 \mathrm{~Hz}$ is not reached even in this case. For the TD and WD models, the spin frequency is not reached.

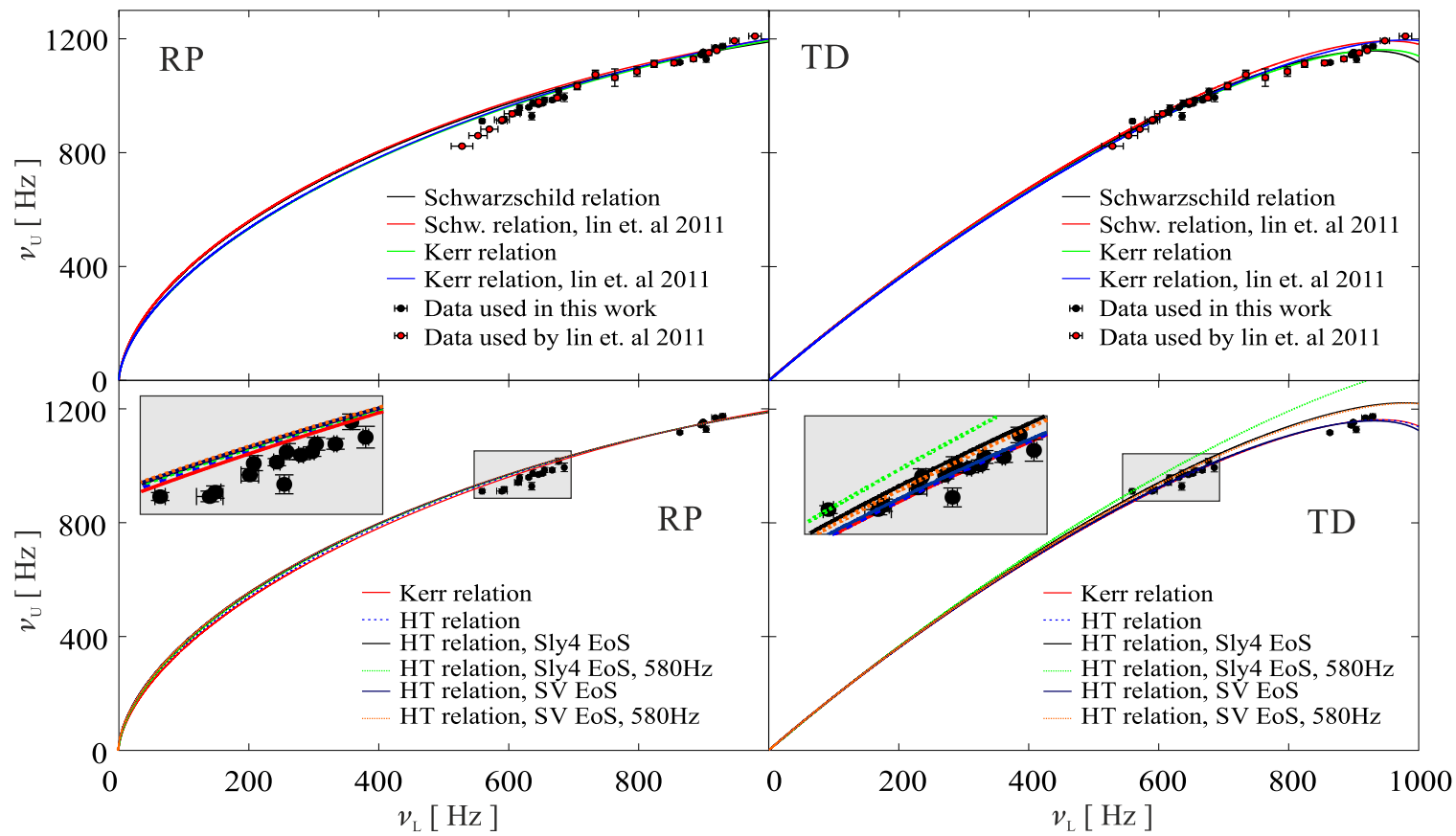

Figure 6. Twin-peak QPO data and examples of their individual fits. Top: best fits of data used in this work (black dots) vs. best fits of data (red dots) used by Lin et al. (2011). Bottom: best fits assuming Kerr spacetime denoted by red lines $\left(\chi_{\min , \mathrm{RP}}^{2}=189 / 20\right.$ d.o.f., $\chi_{\min , \mathrm{TD}}^{2}=122 / 20$ d.o.f). Best fits in Hartle-Thorne spacetimes are denoted by blue lines $\left(\chi_{\min , \mathrm{RP}}^{2}=243 / 19\right.$ d.o.f, $\chi_{\min , \mathrm{TD}}^{2}=123 / 19$ d.o.f.). Best fits in Hartle-Thorne spacetimes restricted to the parametric $2 \mathrm{D}$ surface given by SLy 4 EoS are denoted by black lines $\left(\chi_{\min , \mathrm{RP}}^{2}=303 / 20\right.$ d.o.f, $\chi_{\min , \mathrm{TD}}^{2}=2514 / 20$ d.o.f). Best fits in Hartle-Thorne spacetimes restricted to the parametric $2 \mathrm{D}$ surface given by SV EoS are denoted by dark blue lines $\left(\chi_{\min , \mathrm{RP}}^{2}=327 / 20\right.$ d.o.f, $\chi_{\min , \mathrm{TD}}^{2}=129 / 20$ d.o.f). Best fits in Hartle-Thorne spacetimes restricted by SLy 4 Eos and NS spin $580 \mathrm{~Hz}$ are denoted by green lines $\left(\chi_{\min , \mathrm{RP}}^{2}=305 / 21\right.$ d.o.f, $\chi_{\min , \mathrm{TD}}^{2}=15725 / 21$ d.o.f). Best fits in Hartle-Thorne spacetimes restricted by SV EoS and NS spin $580 \mathrm{~Hz}$ are denoted by orange lines $\left(\chi_{\min , \mathrm{RP}}^{2}=344 / 21\right.$ d.o.f, $\chi_{\min , \mathrm{TD}}^{2}=2233 / 21$ d.o.f $)$. 

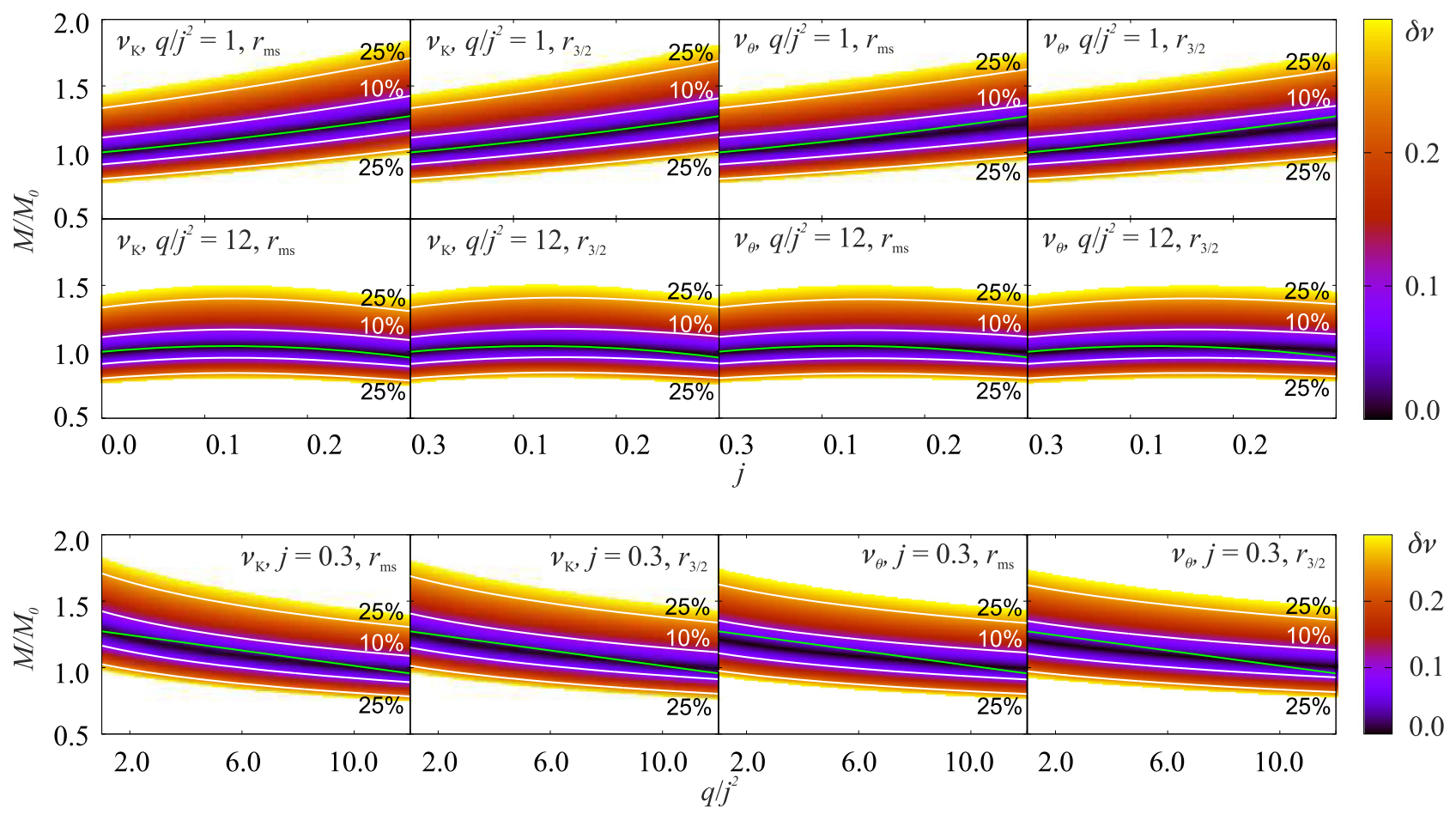

Figure 7. Color-coded maps indicating values of the dimensionless difference between the characteristic frequencies of orbital motion $\delta \nu \equiv\left(\nu\left(M_{0}\right)-\nu(M, j, q)\right) / \nu\left(M_{0}\right)$ calculated assuming the Schwarzschild spacetimes $\left(M=M_{0}\right)$ and Hartle-Thorne spacetimes. Individual panels assume the chosen fixed values of parameters $j$ and $\tilde{q}$. Combinations of parameters indicated by green curves are given by relation $(7), M=M_{0}\left(1+0.7 j+1.02 j^{2}-0.32 q\right)$. Frequencies are calculated at characteristic radii $r_{3: 2}$ and $r_{\mathrm{ms}}$, where the Keplerian and periastron precession frequencies are in a 3:2 and 1:1 ratio.

et al. (2011) is present when we assume Hartle-Thorne as well as Kerr spacetime. There is $\Delta \chi \equiv \sum \operatorname{sign}\left(\chi_{i}\right) \chi_{i}^{2} \sim-150$ for the bottom part of the curve $(i \in\{1 \ldots 14\})$, while it is $\Delta \chi \sim+20$ for the top part of the curve $(i \in\{15 \ldots 22\})$. The possibly required non-geodesic corrections discussed by Török et al. (2012) and Lin et al. (2011) therefore do not depend on the chosen spacetime description (see also Török et al. 2016, in this context). This conclusion is in a good agreement with the suggestion of Török et al. (2012), who implied that the parameters of RP model fits within Hartle-Thorne spacetime should exhibit a degeneracy approximated as

$$
M=M_{0}\left(1+0.7 j+1.02 j^{2}-0.32 q\right),
$$

where $M_{0}=1.78 M_{\odot}$ for $4 \mathrm{U} 1636-53$. This degeneracy is illustrated in Figures 7 and 8, where we also quantify its validity for the other models discussed here.

\subsection{Consideration of Low-frequency QPOs}

Strong restrictions to the model and implied NS mass may be obtained when low-frequency QPOs are considered. This can be clearly illustrated for the RP model, which associates the observed low-frequency QPOs with the Lense-Thirring precession that occurs at the same radii as the periastron precession. Within the framework of the model, the LenseThirring frequency $\nu_{L T}$ represents a sensitive spin indicator (Stella \& Vietri 1998a, 1998b; Morsink \& Stella 1999; Stella et al. 1999). In our previous paper (Török et al. 2012) we carried out a simplified estimate of the underlying NS angular momentum and mass assuming Kerr spacetimes, arriving at values of $j \sim 0.25 \div 0.35$ and $M \sim(2.2 \div 2.4) M_{\odot}$. These values appeared too high when confronted with the

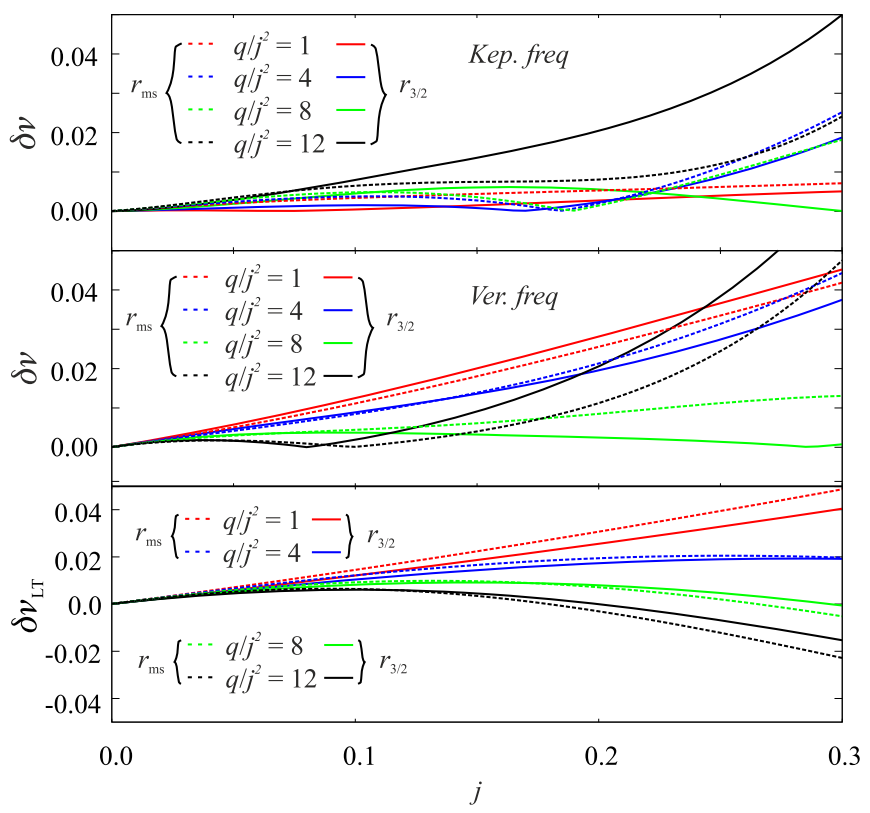

Figure 8. Dimensionless quantity $\delta \nu$ plotted for different values of $q / j^{2}$ and relation (7). We do not include panels for radial and periastron precession frequencies here because the values are the same as for the Keplerian frequency.

implications of the set of $5 \mathrm{EoS}$ assumed within the paper. As discussed here in Section 2, the extended set of EoS can be more compatible with the expectations based on the consideration of Lense-Thirring precession. It is straightforward to extend our previous estimate to Hartle-Thorne spacetime and all $18 \mathrm{EoS}$. The results of such an extension are included in 


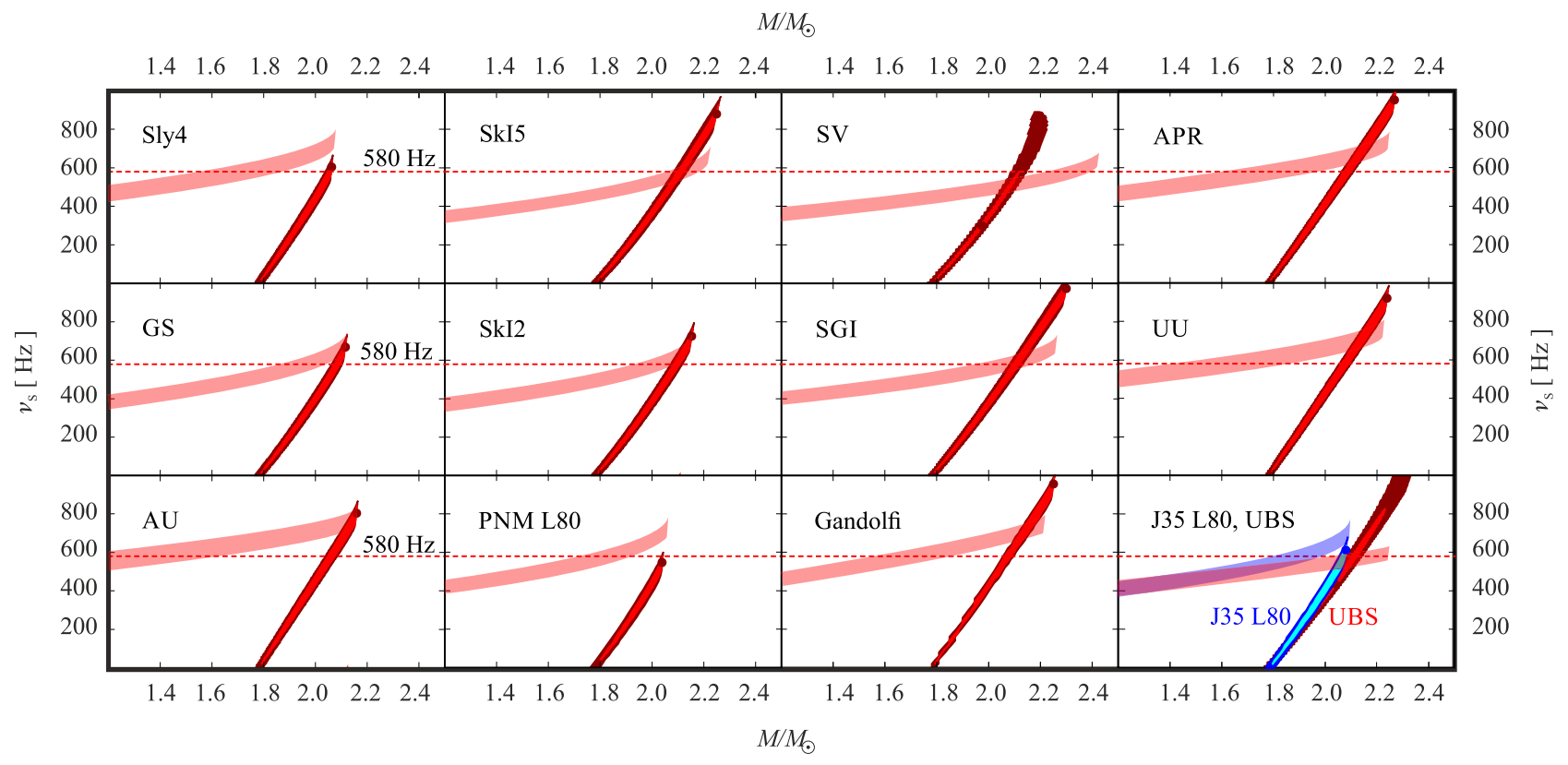

Figure 9. Consideration of RP model assuming both low- and high-frequency QPOs and 13 EoS. The RP model mass-spin maps from Figure 5 are confronted with requirements following from the identification of low-frequency QPOS with the Lense-Thirring precession frequency. The last panel includes the consideration of two different EoS.

Figure 9. We show there $13 \mathrm{EoS}$ that are compatible with the observed twin-peak QPOs and RP model, and demonstrate that 8 of these EoS do not meet the requirements that are based on the consideration of Lense-Thirring preccession. Only 5 EoS are therefore compatible with the model.

We would like to acknowledge the Czech grant GAČR 209/ $12 /$ P740, and internal grants of the Silesian University in Opava, SGS $/ 11,23 / 2013$, SGS $/ 14,15 / 2016$ and IGS/12/ 2015. Z.S. acknowledges the Albert Einstein Center for Gravitation and Astrophysics supported by the Czech Science Foundation grant No. 14-37086G. We are grateful to Marek Abramowicz, Wlodek Kluzniak (CAMK), John Miller (University of Oxford), Will Newton (Texas A\&M UniversityCommerce), Luigi Stella (INAF), and Jiřina Stone (Oak Ridge National Laboratory) for many useful discussions. We also thank to the anonymous referee for the comments and suggestions that greatly helped us to improve the paper. Furthermore, we would like to acknowledge the hospitality of the University of Oxford and the Astronomical Observatory in Rome. Last but not least, we express our sincere thanks to the concierges of the Mlýnská hotel in Uherské Hradiště for their kind help and participation in organizing frequent workshops of the Silesian university and the Astronomical institute.

\section{REFERENCES}

Abramowicz, M. A., Almergren, G. J. E., Kluźniak, W., \& Thampan, A. V. 2003a, arXiv:gr-qc/0312070

Abramowicz, M. A., Bulik, T., Bursa, M., \& Kluźniak, W. 2003b, A\&A, 404, L21

Abramowicz, M. A., Karas, V., Kluźniak, W., Lee, W. H., \& Rebusco, P. 2003c, PASJ, 55, 467

Abramowicz, M. A., \& Kluźniak, W. 2001, A\&A, 374, L19

Agrawal, B. K., Shlomo, S., \& Au, V. K. 2005, PhRvC, 72, 014310

Akmal, A., Pandharipande, V. R., \& Ravenhall, D. G. 1998, PhRvC, 58, 1804

Aliev, A. N., \& Galtsov, D. V. 1981, GReGr, 13, 899

Alpar, M. A., \& Shaham, J. 1985, Natur, 316, 239
Ansorg, M., Kleinwächter, A., \& Meinel, R. 2003, A\&A, 405, 711 Antoniadis, J., Freire, P. C. C., Wex, N., et al. 2013, Sci, 340, 448 Barret, D., \& Boutelier, M. 2008, NewAR, 51, 835

Barret, D., Olive, J.-F., \& Miller, M. C. 2005, MNRAS, 361, 855

Barret, D., Olive, J.-F., \& Miller, M. C. 2006, MNRAS, 370, 1140

Belloni, T., Homan, J., Motta, S., Ratti, E., \& Méndez, M. 2007, MNRAS, 379,247

Belloni, T., Méndez, M., \& Homan, J. 2005, A\&A, 437, 209

Berti, E., White, F., Maniopoulou, A., \& Bruni, M. 2005, MNRAS, 358, 923

Bonazzola, S., Gourgoulhon, E., \& Marck, J.-A. 1998, PhRvD, 58, 104020

Bonazzola, S., Gourgoulhon, E., Salgado, M., \& Marck, J. A. 1993, A\&A, 278, 421

Boshkayev, K., Quevedo, H., Abutalip, M., Kalymova, Z., \& Suleymanova, S. 2015, arXiv:1510.02016

Boutelier, M., Barret, D., Lin, Y., \& Török, G. 2010, MNRAS, 401, 1290

Bursa, M. 2005, in Proc. RAGtime 6/7, Workshops on Black Holes and Neutron Stars, ed. S. Hledík \& Z. Stuchlík (Opava: Silesian Univ.), 39

Čadež, A., Calvani, M., \& Kostić, U. 2008, A\&A, 487, 527

Chandrasekhar, S., \& Miller, J. C. 1974, MNRAS, 167, 63

Demorest, P. B., Pennucci, T., Ransom, S. M., Roberts, M. S. E., \& Hessels, J. W. T. 2010, Natur, 467, 1081

Dutra, M., Louren co, O., Avancini, S. S., et al. 2014, PhRvC, 90, 055203

Dutra, M., Louren co, O., Sá Martins, J. S., et al. 2012, PhRvC, 85, 035201

Galloway, D. K., Muno, M. P., Hartman, J. M., Psaltis, D., \& Chakrabarty, D. 2008, ApJS, 179, 360

Gandolfi, S., Illarionov, A. Y., Fantoni, S., et al. 2010, MNRAS, 404, L35

Germanà, C., Kostić, U., Cadež, A., \& Calvani, M. 2009, in AIP Conf. Ser. 1126, SIMBOL-X: Focusing on the Hard X-Ray Universe, ed. J. Rodriguez \& P. Ferrando (Melville, NY: AIP), 367

Gilfanov, M., Churazov, E., \& Revnivtsev, M. 2000, MNRAS, 316, 923

Glendenning, N. K. 1985, ApJ, 293, 470

Hartle, J. B. 1967, ApJ, 150, 1005

Hartle, J. B., \& Thorne, K. S. 1968, ApJ, 153, 807

Horák, J., Abramowicz, M. A., Kluźniak, W., Rebusco, P., \& Török, G. 2009, A\&A, 499, 535

Kato, S. 2001, PASJ, 53, 1

Kato, S. 2007, PASJ, 59, 451

Kato, S. 2008, PASJ, 60, 111

Klähn, T., Blaschke, D., Sandin, F., et al. 2007, PhLB, 654, 170

Klähn, T., Blaschke, D., Typel, S., et al. 2006, PhRvC, 74, 035802

Kluźniak, W., \& Abramowicz, M. A. 2001, arXiv:astro-ph/0105057

Kluźniak, W., \& Abramowicz, M. A. 2002, arXiv:astro-ph/0203314

Kluźniak, W., Abramowicz, M. A., Kato, S., Lee, W. H., \& Stergioulas, N. 2004, ApJL, 603, L89 
Kluźniak, W., \& Rosińska, D. 2013, MNRAS, 434, 2825

Kostić, U., Čadež, A., Calvani, M., \& Gomboc, A. 2009, A\&A, 496, 307

Lamb, F. K., Shibazaki, N., Alpar, M. A., \& Shaham, J. 1985, Natur, 317, 681

Lin, Y.-F., Boutelier, M., Barret, D., \& Zhang, S.-N. 2011, ApJ, 726, 74

Manko, V. S., Mielke, E. W., \& Sanabria-Gómez, J. D. 2000, PhRvD, 61, 081501

Méndez, M. 2006, MNRAS, 371, 1925

Miller, J. C. 1977, MNRAS, 179, 483

Miller, M. C., Lamb, F. K., \& Psaltis, D. 1998, ApJ, 508, 791

Morsink, S. M., \& Stella, L. 1999, ApJ, 513, 827

Mukhopadhyay, B. 2009, ApJ, 694, 387

Newton, W. G., Gearheart, M., \& Li, B.-A. 2013, ApJS, 204, 9

Nozawa, T., Stergioulas, N., Gourgoulhon, E., \& Eriguchi, Y. 1998, A\&AS, 132,431

Pappas, G. 2015, MNRAS, 454, 4066

Pétri, J. 2005, A\&A, 439, L27

Psaltis, D., Wijnands, R., Homan, J., et al. 1999, ApJ, 520, 763

Rezzolla, L., Yoshida, S., \& Zanotti, O. 2003, MNRAS, 344, 978

Rikovska Stone, J., Guichon, P. A. M., Matevosyan, H. H., \& Thomas, A. W. 2007, NuPhA, 792, 341

Rikovska Stone, J., Miller, J. C., Koncewicz, R., Stevenson, P. D., \& Strayer, M. R. 2003, PhRvC, 68, 034324

Rosińska, D., Kluźniak, W., Stergioulas, N., \& Wiśniewicz, M. 2014, PhRvD, 89, 104001

Steiner, A. W., Gandolfi, S., Fattoyev, F. J., \& Newton, W. G. 2015, PhRvC, 91, 015804

Steiner, A. W., Lattimer, J. M., \& Brown, E. F. 2010, ApJ, 722, 33

Steiner, A. W., Prakash, M., Lattimer, J. M., \& Ellis, P. J. 2005, PhR, 411, 325

Stella, L., \& Vietri, M. 1998a, in Abstracts of the XIX Texas Symp. on Relativistic Astrophysics and Cosmology, ed. J. Paul, T. Montmerle, \& E. Aubourg (Saclay, France: CEA)

Stella, L., \& Vietri, M. 1998b, ApJL, 492, L59

Stella, L., \& Vietri, M. 1999, PhRvL, 82, 17

Stella, L., \& Vietri, M. 2001, in ASP Conf. Ser. 2000, X-ray Astronomy, ed. R. Giacconi, S. Serio, \& L. Stella (San Francisco, CA: ASP), 213
Stella, L., Vietri, M., \& Morsink, S. M. 1999, ApJL, 524, L63

Stergioulas, N., \& Friedman, J. L. 1995, ApJ, 444, 306

Strohmayer, T. E., \& Markwardt, C. B. 2002, ApJ, 577, 337

Stuchlík, Z., Konar, S., Miller, J. C., \& Hledík, S. 2008, A\&A, 489, 963

Stuchlík, Z., Kotrlová, A., \& Török, G. 2013, A\&A, 552, A10

Stuchlík, Z., Kotrlová, A., Török, G., \& Goluchová, K. 2014, AcA, 64, 45

Stuchlík, Z., Urbanec, M., Kotrlová, A., Török, G., \& Goluchová, K. 2015 AcA, 65, 169

Stute, M., \& Camenzind, M. 2002, MNRAS, 336, 831

Titarchuk, L., \& Wood, K. 2002, ApJL, 577, L23

Török, G. 2009, A\&A, 497, 661

Török, G., Abramowicz, M. A., Bakala, P., et al. 2008a, AcA, 58, 15

Török, G., Abramowicz, M. A., Bakala, P., et al. 2008b, AcA, 58, 113

Török, G., Bakala, P., Stuchlik, Z., \& Čech, P. 2008c, AcA, 58, 1

Török, G., Bakala, P., Šrámková, E., et al. 2012, ApJ, 760, 138

Török, G., Bakala, P., Šrámková, E., Stuchlík, Z., \& Urbanec, M. 2010, ApJ, 714,748

Török, G., Goluchová, K., Horák, J., et al. 2016, MNRAS, 457, L19

Török, G., \& Stuchlík, Z. 2005, A\&A, 437, 775

Török, G., Stuchlík, Z., \& Bakala, P. 2007, CEJPh, 5, 457

Török, G., Urbanec, M., Adámek, K., \& Urbancová, G. 2014, A\&A, 564, L5

Urbanec, M., Běták, E., \& Stuchlík, Z. 2010a, AcA, 60, 149

Urbanec, M., Miller, J. C., \& Stuchlík, Z. 2013, MNRAS, 433, 1903

Urbanec, M., Török, G., Šrámková, E., et al. 2010b, A\&A, 522, A72

van der Klis, M. 2005, AN, 326, 798

van der Klis, M. 2006, in Compact stellar X-ray sources, ed. W. Lewin \& M. van der Klis (Cambridge: Cambridge Univ. Press), 39

Wagoner, R. V. 1999, PhR, 311, 259

Wagoner, R. V., Silbergleit, A. S., \& Ortega-Rodríguez, M. 2001, ApJL, 559, L25

Wang, D. H., Chen, L., Zhang, C. M., et al. 2015, MNRAS, 454, 1231

Watts, A. L. 2012, ARA\&A, 50, 609

Wiringa, R. B., Fiks, V., \& Fabrocini, A. 1988, PhRvC, 38, 1010

Zhang, C.-M. 2005, ChJAS, 5, 21 